\title{
EDITORIAL
}

\section{Treatment of myelodysplastic syndromes with exogenous erythropoietin: a new therapeutic paradigm}

Leukemia (2006) 20, 1481-1483. doi:10.1038/sj.leu.2404291

In the last 25 years, significant advances have occurred in the diagnostic and prognostic classifications of myelodysplastic syndromes (MDS); however, the correct choice of therapy continues to be an enormous challenge. The mainstay of therapy for MDS has been supportive care. The only available option with a probability of cure in a minority of MDS patients is stem cell transplant. ${ }^{1}$ Variable multilineage peripheral cytopenia, the major clinical feature of MDS, provides rationale for the use of exogenous growth factors. With refractory anemia being the clinical hallmark of MDS, erythropoietin (EPO) is widely used in the treatment of MDS. No clear deficiency of endogenous serum erythropoietin (sEPO) can be consistently detected in MDS patients and, in fact, there is considerable variability in patients with the same degree of anemia. Often, inappropriately low levels of sEPO are noted in severely anemic patients. ${ }^{2}$ As a result, exogenous administration of recombinant human EPO was instituted as a therapy for MDS in early 1990s. Subsequently, a number of studies have established its utility, especially in low- to intermediate-1-risk categories of MDS per International Prognostic Scoring System (IPSS).

A significant refinement has occurred in the ability to use EPO therapy over the last 15 years with erythroid response rates exceeding $50 \%$ in select groups of low-risk MDS patients. ${ }^{3,4}$ As reviewed by Clavio et al., ${ }^{3}$ initially EPO was used in all FrenchAmerican-British (FAB) classification subgroups with an overall erythroid response rate of $\sim 20 \%$. Subsequently, with the addition of granulocyte colony-stimulating factor (GCSF) to $\mathrm{EPO}$, the response rates appear to be higher with $\sim 40 \%$ overall erythroid responses primarily in low- to intermediate-1-risk patients. ${ }^{3}$ Hellström-Lindberg and co-workers proposed a model with a scoring system to predict response to EPO therapy based on the pretreatment packed red blood cell transfusion requirement and sEPO levels. In line with this predictive model, in a recent long-term follow-up of three major Nordic studies, the patients selected for low transfusion requirements and low sEPO were found to show an overall erythroid response rate of $60 \%$ with EPO alfa treatment. ${ }^{4}$ Another erythropoietic agent called darbepoietin alfa (Aranesp) also was found to elicit $\sim 45 \%$ erythroid response in a similar selected MDS patient subgroup. ${ }^{5}$ Presently, thus, via patient selection and via addition of GCSF as needed the erythroid response rates to EPO therapy in MDS have improved to a level of $\sim 50-60 \%$. Further advancement in this treatment modality can only be expected by gaining additional insights into the process of impairment of erythropoiesis in these patients.

\section{Dysregulation of erythropoiesis in MDS}

Dyserythropoiesis is a primary pathologic feature of all subgroups of MDS patients, and available data provide some important clues for improving the therapeutic outcome in general, and for EPO therapy in particular. Normal erythropoiesis occurs in discrete niches within bone marrow, usually containing a macrophage in the center surrounded by proliferating and differentiating immature erythropoietic progenitors. The erythropoiesis cell unit appears to autoregulate its expansion capacity. Fas-mediated apoptosis has been shown to regulate the erythroid island expansion. ${ }^{6,7}$ Immature proliferating erythroid progenitors express high amounts of Fas. The expression of Fas-ligand (Fas-L) gradually increases as the cells mature and differentiate. It is thought that the crosslinking of Fas-L on the differentiating cells with Fas on more immature proliferating cells induces apoptosis in the latter and controls erythropoiesis. ${ }^{6}$ Furthermore, EPO levels in the marrow may determine the sensitivity of immature erythroid progenitors to Fas-induced apoptosis, and that erythropoiesis may be regulated by an appropriate balance between EPO levels in the erythroid niche and the levels of Fas-L expression by maturing erythroid progenitors. Additionally, the interplay between EPO and Fas/ Fas- $\mathrm{L}$ appears to be under an extracellular autocrine/paracrine influence of a stoichiometric balance of different cytokines. The predominant positive regulator, undoubtedly, is EPO. On the other hand, a number of proinflammatory cytokines like tumor necrosis factor alpha (TNF $\alpha$ ), interleukin 1 beta (IL1 $\beta)$, interferon gamma and transforming growth factor beta exert a negative regulation on erythropoiesis. ${ }^{8-11}$ Whereas all these negative regulators are increased in concentration in MDS marrows in general, the one that seems to correlate with many pathologic as well as clinical features of MDS is TNF $\alpha$.

Many studies including ours have reported increased levels of $\mathrm{TNF} \alpha$ in the serum as well as in the bone marrows of the majority of MDS patients and has been thought to be a key player in the pathobiology of MDS. ${ }^{12,13}$ TNF $\alpha$ on the one hand may upregulate Fas- $\mathrm{L}$ in erythroid progenitors inducing apoptosis in these cells. ${ }^{14}$ Whereas on the other hand, it could transcriptionally inhibit EPO receptor (EPO-R), interfere with EPO signaling and affect the survival of erythroid progenitors. In MDS-derived erythroid progenitors, lower levels of EPO-R moieties per cell, and a lack of EPO-activated, differentiationrelated increase in GATA-1 transcription factor, have been shown in the past. ${ }^{15,16}$ Therefore, it appears that in MDS marrow, TNF $\alpha$ may actively induce apoptosis and, in addition, suppress EPO-mediated survival signals.

\section{TNF $\alpha$ as a determinant of erythroid response to MDS therapy}

The aforementioned observations are corroborated by clinical findings from studies using EPO treatment and those using TNF suppression strategies. In two separate trials using EPO monotherapy for MDS, it was evident that patients with elevated serum TNF $\alpha$ levels (above normal range, i.e. $>($ mean $+2 \times$ s.d.) for healthy donors) did not respond to EPO therapy. ${ }^{17,18}$ Both studies used weight-based EPO regimens with a total weekly dose ranging from 30000 to $>70000 \mathrm{U}$ in unselected MDS patients and used response criteria parallel to the International 
Working Group criteria (IWG criteria). It may be noted that a lack of response in these studies was determined only after a chance for dose escalation.

In another regimen where EPO + GCSF were combined with all-trans-retinoic acid (ATRA) and alpha tocopherol, nonresponders not only had significantly higher levels of serum $\mathrm{TNF} \alpha$ before therapy but also the TNF $\alpha$ levels continued to rise during treatment in these patients. ${ }^{19}$ Similarly, during cytokinesuppressing therapy with pentoxifylline + ciprofloxacine \pm decadron, the responses were noted only in those MDS patients who showed continued reduction in bone marrow $\mathrm{TNF} \alpha$ levels. ${ }^{20}$ Furthermore, a direct suppression of TNF $\alpha$ with infliximab, a chimeric anti-TNF $\alpha$ antibody in an anecdotal study with two low-risk MDS patients having elevated serum $\mathrm{TNF} \alpha$, resulted in encouraging erythroid response in both subjects. ${ }^{21}$ Obviously, although TNF $\alpha$ may be a key component in the pathogenesis of MDS, mere suppression of TNF $\alpha$ may not be therapeutically sufficient in all patients. Yet, such an approach may be essential in considering any future combinatorial therapeutic approach as described below.

\section{Proposal for new therapeutic paradigm}

The treatment model for MDS proposed here initially stratifies patients by serum TNF $\alpha$ levels. Subsequently, different sequences of exogenous $\mathrm{EPO} \pm \mathrm{GCSF}$, anti-TNF $\alpha$ agents and/or cytotoxic modalities are suggested for the treatment of different MDS groups identified by the present model.

The studies on the pathobiology of MDS appear to indicate three prominent features that may have significant therapeutic implications in these disorders:

1. Elevated levels of proinflammatory cytokines, particularly TNF $\alpha$.

2. Excessive intramedullary apoptosis and impairment of hematopoietic cell maturation.

3. Presence of immature blasts.

In the low- to intermediate-1-risk population, the first two features form the basis of the therapeutic strategy, whereas in the high-risk group, the rapidly expanding population of blasts needs to be targeted. Except for a highly selected group of patients where single-agent therapy can suffice, a combination of strategies is desirable for the majority of MDS patients. With anemia being a common feature of all MDS subtypes, growth factors may be of benefit beyond their current use in low- to intermediate-1-risk population. They will have to be combined with other agents, with a proper biologic rationale and sequence, as indicated in the proposed therapeutic paradigm in Figure 1. So far, significantly high response rates are predictable only in MDS patients with chromosome 5 abnormalities being treated with lenalidomide.

For patients not showing chromosome 5 abnormalities, we propose that, if low/intermediate-1-risk and intermediate-2/ high-risk groups are further stratified by their serum $\mathrm{TNF} \alpha$ levels, there may be a distinct therapeutic advantage. The high sTNF $\alpha$ group in low/intermediate-1-risk category may be first treated with an anti-TNF $\alpha$ agent like infliximab or lenalidomide followed by EPO \pm GCSF. On the other hand, in intermediate-2/ high-risk group with elevated $\operatorname{sTNF} \alpha$, control of immature blasts with agents such as the farnesyl transferase inhibitors, azacytidine or arsenic trioxide, may be employed sequentially or concurrently with an anti-TNF $\alpha$ agent followed by EPO \pm GCSF. Further, the low sTNF $\alpha$ group in low/intermediate- 1 category may respond well to EPO alone with GCSF as needed, whereas the same group in intermediate-2/high-risk category may need cytotoxic treatment followed by $\mathrm{EPO} \pm \mathrm{GCSF}$ as needed. Although EPO has been combined safely with other agents in the past including GCSF, granulocyte macrophage colonystimulating factor, IL3, ATRA, amifostine and thalidomide, the newly described erythropoietic factor, darbepoietin alfa, when combined with thalidomide in low/intermediate-1-risk MDS patients showed high incidence of thromboembolic events. $^{3,22,23}$ Thus, caution needs to be exerted while combining specific growth factor with other therapies.

Clearly, with the limited success of single-agent therapies for MDS, save lenalidomide in a subset of patients with chromosome 5 abnormalities, the hope for future therapeutic success in these disorders lies in further defining biologic subsets of

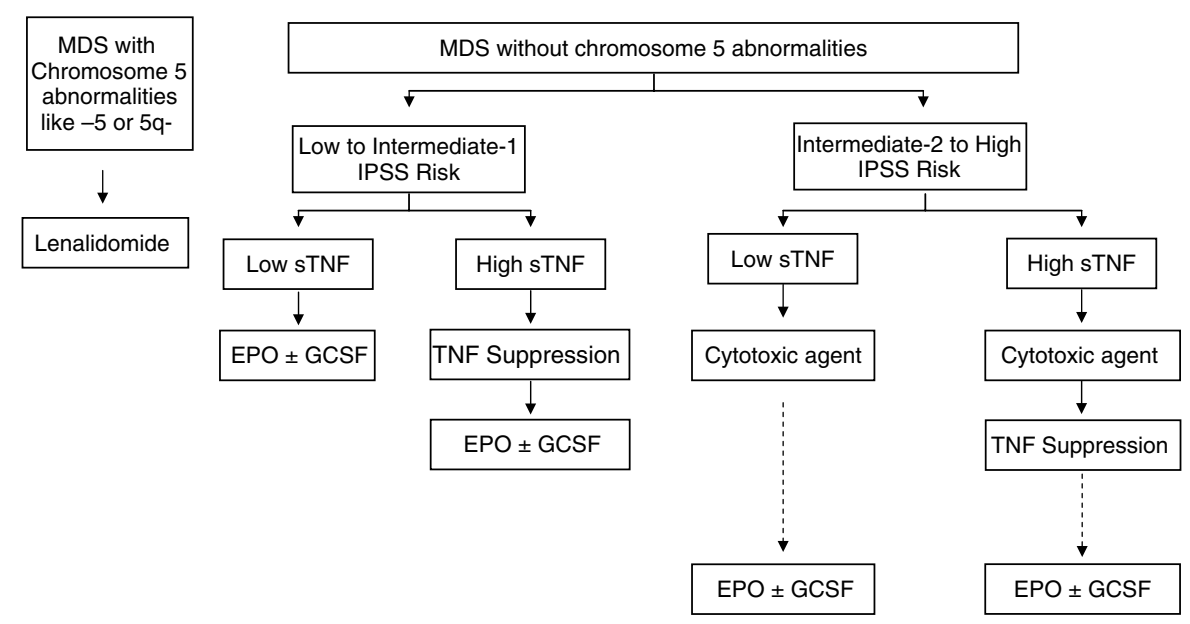

Figure 1 Proposed model for stratification of MDS patients other than those with chromosome 5 abnormalities, based on serum TNF $\alpha$ (sTNF $\alpha$ ) levels. While in low/intermediate-1-risk category, EPO \pm GCSF could suffice for patients with low sTNF $\alpha$, a sequential combination of TNF $\alpha$ suppression with $\mathrm{EPO} \pm$ GCSF may be needed for the patients having high sTNF $\alpha$ levels. In the intermediate-2/high-risk category, a cytotoxic agent could be used in patients with low levels of sTNF $\alpha$, whereas in those with high sTNF $\alpha$, cytotoxic agent may be used in combination with TNF $\alpha$ suppression. In the intermediate-2/high-risk group, EPO \pm GCSF may be used as needed (dotted arrow). It may be noted that, owing to a proven efficacy of lenalidomide in patients with chromosome 5 abnormalities, the choice of treatment in such patients could be single-agent lenalidomide. 
patients and using combination approaches. Our model attempts to outline therapeutic principles with a biologic rationale. Safety and efficacy of the specific combinations will need to be evaluated as we look to further improve the therapeutic outcome of growth factor treatment for MDS.

SD Mundle ${ }^{1,2}$ and A Raza ${ }^{3}$

${ }^{1}$ OrthoBiotech Inc., Bridgewater, NJ, USA;

${ }^{2}$ Rush University Medical Center, Chicago, IL, USA and

${ }^{3}$ University of Massachusetts Medical School, Worcester, MA,

USA

E-mail: smundle@obius.jnj.com

\section{References}

1 Steensma DP, Bennett JM. The myelodysplastic syndromes: diagnosis and treatment. Mayo Clin Proc 2006; 81: 104-130.

2 Aul C, Arnin M, Runde V, Schneider W. Serum erythropoietin concentrations in patients with myelodysplastic syndromes. Leukemia Res 1991; 15: 571-575.

3 Clavio M, Balleari B, Garrone A, Ballerina F, Varaldo R, Michelis $\mathrm{GL}$ et al. Haematopoietic growth factors in myelodysplastic syndromes: toward patient-oriented therapy? J Exp Clin Cancer Res 2005; 24: 5-16.

4 Jädersten M, Montgomery SM, Dybedal I, Porwit-MacDonald A, Hellström-Lindberg E. Long-term outcome of treatment of anemia in MDS with erythropoietin and G-CSF. Blood 2005; 106: $803-811$.

5 Stasi R, Abruzzese E, Lanzetta G, Terzoli E, Amadori S. Darbepoetin alfa for the treatment of anemic patients with lowand intermediate-1-risk myelodysplastic syndromes. Ann Oncol 2005; 16: 1921-1927.

6 De Maria R, Testa U, Luchetti L, Stassi SE, Felli RN, Samoggia P et al. Apoptotic role of Fas/Fas ligand system in the regulation of erythropoiesis. Blood 1999; 93: 796-803.

7 Claessens Y-E, Park S, Dubart-Kupperschmitt A, Mariot V, Garrido $\mathrm{C}$, Chrétien $\mathrm{S}$ et al. Rescue of early-stage myelodysplastic syndrome-deriving erythroid precursors by the ectopic expression of a dominant-negative form of FADD. Blood 2005; 105: 4035-4042.

8 Johnson RA, Waddelow TA, Caro J, Oliff A, Roodman GD. Chronic exposure to tumor necrosis factor in vivo preferentially inhibits erythropoiesis in nude mice. Blood 1989; $\mathbf{7 4}$ 130-138.

9 Jacobs-Helber SM, Roh K-H, Bailey D, Sessypris EN, Ryan IJ, Chen J et al. Tumor necrosis factor-alpha expressed constitutively in erythroid cells or induced by erythropoietin has negative and stimulatory roles in normal erythropoiesis and erythroleukemia. Blood 2003; 101: 524-531.

10 Chuncharunee S, Carter CD, Studtmann KE, Caro J, Coffey RJ, Dessypris EN. Chronic administration of transforming growth factor-beta suppresses erythropoietin-dependent erythropoiesis and induces tumor necrosis factor in vivo. Br J Haematol 1993; 84: 374-380.

11 Means Jr RT, Dessypris EN, Krantz SB. Inhibition of human erythroid colony-forming units by interleukin- 1 is mediated by gamma interferon. J Cell Physiol 1992; 150: 59-64.

12 Verhoef GE, De Schouwer P, Ceuppens JL, Van Damme J, Goossens W, Boogaerts MA. Measurement of serum cytokine levels in patients with myelodysplastic syndromes. Leukemia 1992; 6: 1268-1272.

13 Shetty V, Mundle S, Alvi S, Showel M, Broady-Robinson L, Dar S et al. Measurement of apoptosis, proliferation and three cytokines in 46 patients with myelodysplastic syndromes. Leukemia Res 1996; 20: 891-900.

14 Otsuki T, Nagakura S, Wang J, Bloom M, Grompe M, Liu JM. Tumor necrosis factor-alpha and CD95 ligation suppress erythropoiesis in Fanconi anemia C gene knockout mice. Cell Physiol 1999; 179: 79-86.

15 Takeshita A, Shinjo K, Naito K, Ohnishi K, Higuchi M, Ohno R. Erythropoietin receptor in myelodysplastic syndrome and leukemia. Leukemia Lymphoma 2002; 43: 261-264.

16 Tehranchi R, Invernizzi R, Grandien A, Zhivotovsky B, Fadeel B, Forsblom A-M et al. Aberrant mitochondrial iron distribution and maturation arrest characterize early erythroid precursors in low-risk myelodysplastic syndromes. Blood 2005; 106: 247-253.

17 Musto P, Matera R, Minervini MM, Checchia-de Ambrosio C, Bodenizza C, Falcone A et al. Low serum levels of tumor necrosis factor and interleukin- $1 \beta$ in myelodysplastic syndromes responsive to recombinant erythropoietin. Haematologica 1994; 79: 265-268.

18 Stasi R, Brunetti M, Bussa S, Conforti M, Martin LS, La Presa M et al. Serum levels of tumor necrosis factor- $\alpha$ predict response to recombinant human erythropoietin in patients with myelodysplastic syndrome. Clin Lab Haematol 1997; 19: 197-201.

19 Ganser A, Maurer A, Contzen C, Seipelt G, Ottmann OG, Schadeck-Gressel C et al. Improved multilineage response of hematopoiesis in patients with myelodysplastic syndromes to a combination therapy with all-trans-retinoic acid, granulocyte colony-stimulating factor, erythropoietin and alpha-tocopherol. Ann Hematol 1996; 72: 237-244.

20 Reza S, Shetty V, Dar S, Qawi H, Raza A. Tumor necrosis factoralpha levels decrease with anticytokine therapy in patients with myelodysplastic syndromes. J Interferon Cytokine Res 1998; 18: 871-877.

21 Stasi R, Amadori S. Infliximab chimeric anti-tumor necrosis factor alpha monoclonal antibody treatment for patients with myelodysplastic syndromes. Br J Haematol 2002; 116: 334-337.

22 Musto P, Falcone A, Sanpaolo G, Bodenizza C. Combination of erythropoietin and thalidomide for the treatment of anemia in patients with myelodysplastic syndromes. Leukemia Res 2005; 30: 385-388.

23 Steurer M, Sudmeier I, Stauder R, Gastl G. Thromboembolic events in patients with myelodysplastic syndromes receiving thalidomide in combination with darbepoietin-alpha. Br J Haematol 2003; 121 : 101-103. 